\title{
Investigation of the Kapitza Anomaly by Frequency-Resolved Phonon Transport in Silicon Wafers
}

\author{
Wolfgang Klar and Kurt Laßmann \\ 1. Physikalisches Institut, Pfaffenwaldring 57, D-7000 Stuttgart 80, FRG
}

The threshold at $85 \mathrm{GHz}$ for anomalously large transmission of phonons into LHe has been investigated with Si-wafers for various surface treatments and coverages by phonon spectroscopy. With this multiple boundary scattering geometry we observe an increased sensitivity to spectral features. Connected with the threshold there is a phase shift of the signal compatible with a loss of the slower diffuse portion of the phonon signal to the covering liquid helium. This phase shift depends nonlinearly on modulation frequency between $5 \mathrm{kHz}$ and $200 \mathrm{kHz}$ (modulation of the generator to sort out the monochromatic phonons) and in a characteristic manner on the surface treatment. The finite phase shift at $5 \mathrm{kHz}$ is indicative of very long lived $85 \mathrm{GHz}$ phonons up into the $100 \mu \mathrm{s}$ range inspite of the multiple Si-LHe-interface scattering involved. Minor differences in polishing quality show up clearly in the phase shift at low modulation frequencies.

Phonon spectroscopy with superconducting tunneljunctions covers an interesting range of energies (of the order of $\mathrm{meV}$ ) and wavelengths (of the order of $10 \mathrm{~nm}$ ) rendering it a sensitive probe of energetic or geometric properties of surfaces and interfaces in the corresponding ranges. This has been shown by a number of experiments investigating diffuse and specular, elastic and inelastic phonon scattering. (For a recent review, see [1].) In these experiments the samples were large enough to allow time and space resolved analysis of single scattering events.

There are, however, good reasons to study the surface and interface scattering in thin planar structures, namely their growing technological importance as well as the increased sensitivity due to multiple phonon scattering.

We show in this contribution that small differences in surface quality of silicon wafers show up in the phonon scattering signal. In particular, we find a phase shift of the modulated signal at a phonon frequency of $85 \mathrm{GHz}$ corresponding to the threshold for anomalous transmission of diffusely scattered phonons into liquid helium first reported by Koblinger et al [2]. This phase shift depends on modulation frequency in a way characteristic for the surface quality as will be shown below.

The configuration of our set-up is as follows: Pairs of Sn-junction generators (emitted frequencies 0 to $280 \mathrm{GHz}$ as determined by junction bias) and Al-junction detectors (detection thresholds between $\sim 70 \mathrm{GHz}$ and $\sim 100 \mathrm{GHz}$ depending on gap width with some bolometric detection below threshold) are evaporated onto one side of a $20 \mathrm{~mm} \times 20 \mathrm{~mm}$, $.44 \mathrm{~mm}$ thick, (111)-oriented Si-Wafer to allow two different propagation distances (4 $\mathrm{mm}$ and $11 \mathrm{~mm}$ ) and orientations (equivalent [ī10] and [112]]). Junction areas have $1 \mathrm{~mm} \phi_{;}$the contact regions to the junctions do not interfere with the propagation paths.

A typical frequency (i.e. generator bias or current) dependence of the detector signal is shown in Fig. 1 (Upper curve): Between A and B the signal is made up by $280 \mathrm{GHz}$ Sn recombination phonons. Between $B$ and $C$ relaxation phonons with frequencies smaller than the detector threshold $(\sim 65 \mathrm{GHz})$ are detected bolometrically. Shortly after the signal rise at threshold $\mathrm{C}$ there is a decay starting at about $85 \mathrm{GHz}$ with a concomitant phase shift on the quadrature $\left(90^{\circ}\right)$ channel of the Vector-Lock-In corresponding to a faster signal arrival (Lower

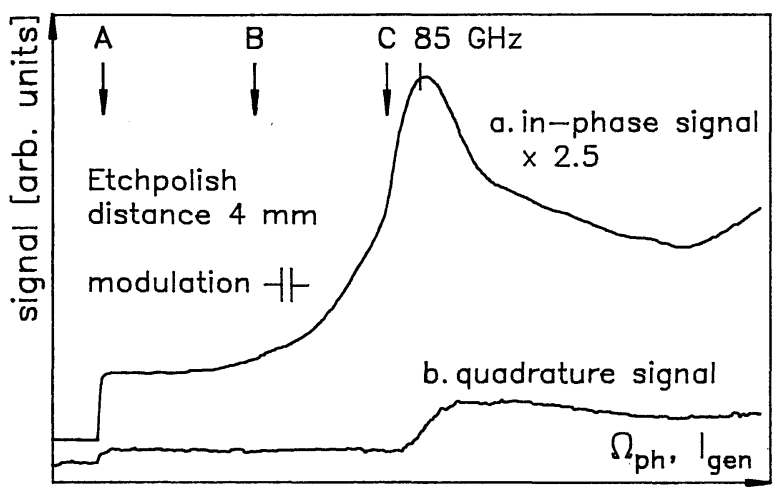

Fig. 1. Typical dependence of the detector signal on modulated generator current I which can be calibrated in phonon frequency $\Omega_{\mathrm{ph}}^{\mathrm{gen}}$. A, B, C see text. Wafer in contact with LHe.

The phase shift at about $85 \mathrm{GHz}$ is determined for horizontal quadrature signal before and after the shift. The in-phase signal is then maximal.

curve). From ballistic single scattering experiments it is known that the anomalous transmission is due to diffusely scattered phonons the loss of which causes the decay [1]. The assumption then that the specularly scattered phonons arrive faster in the average is compatible with the observed sign of the phase shift.

In Figs. 2-4 we show the dependence of this phase shift on modulation frequency for different experimental conditions. Fig. 2 shows that there is already a distinct difference between a very good Syton polish and an optimum quality furnished by Wacker Chemitronic. It is seen that even at modulation frequencies as low as $5 \mathrm{kHz}$ the phase shift is of about the same magnitude as at the highest modulation frequency of $200 \mathrm{kHz}$. In contrast, a $25 \mu \mathrm{m}$ diamond polished surface shows a small phase shift at small modulation frequencies rising to large values at the upper end of the range. (Fig. 3) Repeatedly wiping of residual dust particles with the rim of a acetone-wetted lens-tissue results in a degradation of a Syton polished surface to the quality of diamond polish (Fig. 3). In all cases there is no significant difference on propagation direction. The phase shift is larger for the longer distance, the difference increasing from the lower to the upper end of modulation frequencies (Fig. 4). The double maximum structure of the phase shift 


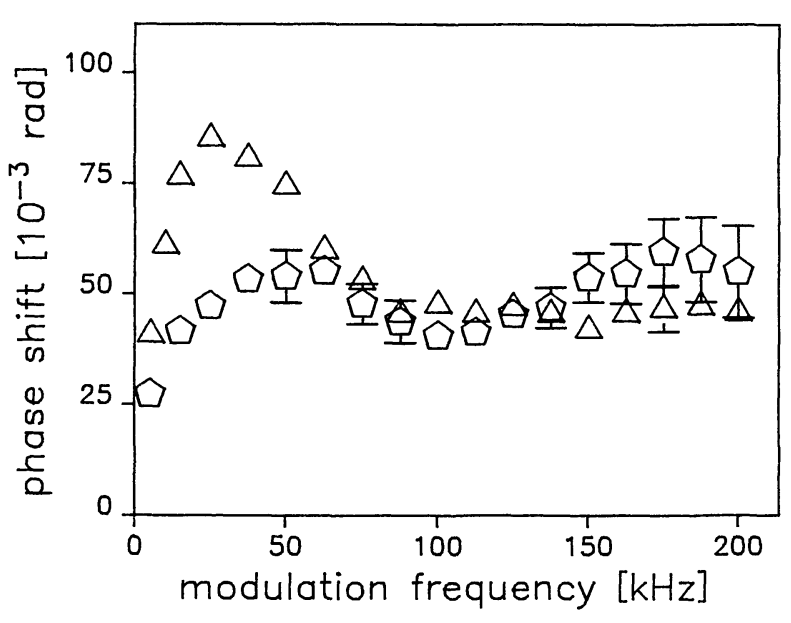

Fig. 2. Dependence of the phase shift on modulation frequency for highly polished surfaces. Triangles: optimum polish furnished by Wacker Chemitronic, pentagons: Syton polish

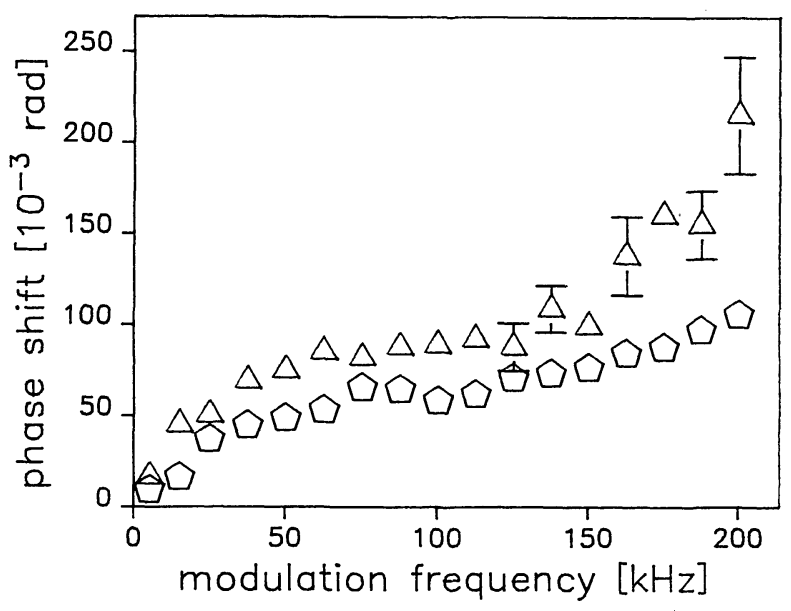

Fig. 3. Dependence of the phase shift on modulation frequency for degraded surfaces. Triangles: $25 \mu \mathrm{m}$ diamond polish, pentagons: degraded Syton polish by repeated wiping with lens-tissue

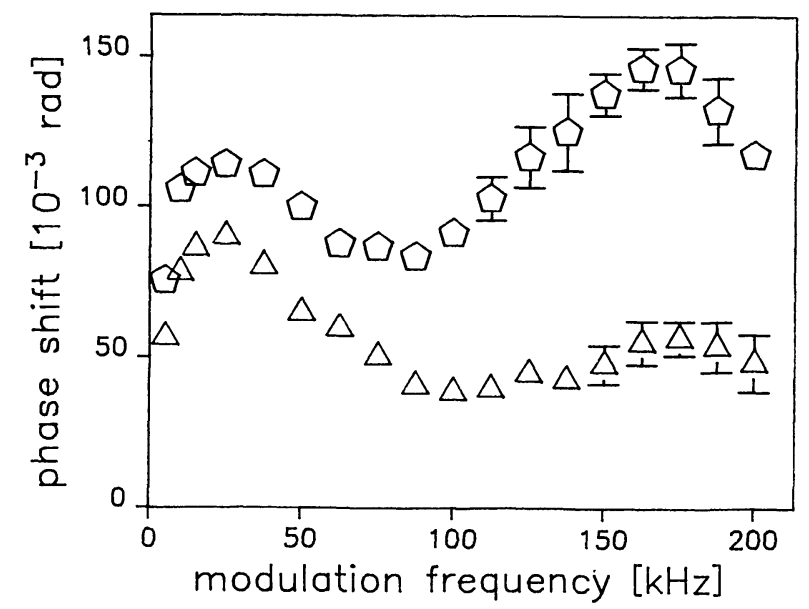

Fig. 4. Dependence of the phase shift on modulation frequency for different distances. Triangles: $4 \mathrm{~mm}$, pentagons: $11 \mathrm{~mm}$. dependence on modulation frequency cannot be an artefact of the sampling theorem since it depends on experimental conditions with the lower maximum being about a factor of seven below the upper maximum. Instead, it seems that the large changes with surface conditions seen at small modulation frequencies indicate the big influence on long-lived phonons in the $100 \mu$ s range. The in-phase signal i.e. the signal decay after the Kapitza threshold, does not show such large differences; presumably, because much of the signal reduction is due to transmission of phonons through the rough surface of the generator junction itself. It is not easy to decouple the junctions alone and loss-free from contact to the helium because of the small dimensions involved. A coverage of the whole wafer by a thin absorbing/reflecting oil film shows a decoupling effect removing the Kapitza decay completely. However, the signal is also much reduced by a frequency independent absorption in the oil so that the phase shift in this case was not measurable. Some dependence of the in-phase signal on surface quality can be observed: A small structure at about $130 \mathrm{GHz}$ and beyond this value a further decay are more distinct for high than for low quality surfaces.

Because of the problem of temperature stability it was not yet possible to do modulated cw-measurements in vacuum to see whether there is also a phase shift at $85 \mathrm{GHz}$. This would then mean a sudden change in the surface scattering mechanism which could be due to resonant states of the surface. If no phase shift will be seen this would mean that the anomalous transmission is due to a combination effect: points of diffuse scattering form radiating centers together with the covering helium. So far, we have only succeeded to compare time (but not frequency) resolved measurements in vacuum and in helium. In vacuum we find a steep rise after the time for ballistic specular reflection propagation peaking narrowly and followed by a broader maximum with diffusion-like tail. This latter portion disappears with helium contact whereas much of the first signal peak remains which is compatible with the assumption that it is made up mainly of the faster specular part of the signal.

Though our results are not yet conclusive the presented method is a new and promising means to investigate the problem of anomalous transmission of high frequency phonons into liquid helium and also problems connected with properties of interfaces and surfaces of planar structures.

This work was supported by the Deutsche Forschungsgemeinschaft. This support and also useful discussions with W. Eisenmenger, M. Gienger, S. Burger, and P.O. Hahn (who kindly helped us with highly polished wafers) are gratefully acknowledged.

\section{REFERENCES}

1) W. Eisenmenger in: Phonon Scattering in Condensed Matter V, Springer Series in Solid State Sciences 68 (1986) ed. by A. C. Anderson and J. P. Wolfe, p. 204

2) O. Koblinger, K. Heim, M. Welte, and

W. Eisenmenger,

Phys. Rev. Lett. 51(1983) 284 